\title{
The impact of affect-aware support on learning tasks that differ in their cognitive demands
}

\author{
Beate Grawemeyer ${ }^{1}$, Manolis Mavrikis ${ }^{2}$, \\ Claudia Mazziotti ${ }^{3}$, Anouschka van Leeuwen ${ }^{4}$, and Nikol Rummel ${ }^{3}$ \\ 1 School of Computing, Electronics and Maths, Coventry University, UK \\ ac7655@coventry.ac.uk, \\ 2 UCL Knowledge Lab, Institute of Education, University College London, UK \\ m.mavrikis@ioe.ac.uk, \\ 3 Institute of Educational Research, Ruhr-Universität Bochum, DE \\ claudia.mazziotti@rub.de, nikol.rummel@rub.de \\ 4 Faculty of Social and Behavioral Science, Utrecht University, NL \\ A.vanLeeuwen@uu.nl
}

\begin{abstract}
This paper investigates the effect of affect-aware support on learning tasks that differ in their cognitive demands. We conducted a study with the iTalk2learn platform where students are undertaking fractions tasks of varying difficulty and assigned in one of two groups; one group used the iTalk2learn platform that included the affect-aware support, whereas in the other group the affect-aware support was switched off and support was provided based on students' performance only. We investigated the hypothesis that affect-aware support has a more pronounced effect when the cognitive demands of the tasks are higher. The results suggest that students that undertook the more challenging tasks were significantly more in-flow and less confused in the group where affect-aware support was provided than students who were supported based on their performance only.
\end{abstract}

\section{Introduction}

It is well understood that not only cognitive factors but also students' affective states play a major role for learning. In recent years some studies have been undertaken to shed more light on the relation between learning and emotion [4, $2,1]$. However, previous research has missed investigation of how characteristics of learning tasks (e.g., difficulty level) are linked to students' affective states. Therefore, the first goal of our study is to investigate whether and to what extent students differ in their emotional response to different kinds of tasks. Since we know from $[6,2]$ research and our own research that affect-aware support has the power to promote or keep students in a 'positive' affective state, the second goal of our study is to investigate what role the affect aware support plays when students engage with different kinds of tasks.

We report results from a user study which investigated the hypothesis that affect-aware support has a more pronounced effect when the cognitive demands of the task are higher. In Grawemeyer et al. [3] we describe the development of 
affect-aware support and the effect of the support across fraction learning tasks. In contrast, in this paper we report on the impact of affect-aware support on tasks that differ in their cognitive demands.

\section{The iTalk2Learn platform}

iTalk2learn is a learning platform for children aged 8-12 years old who are learning fractions.

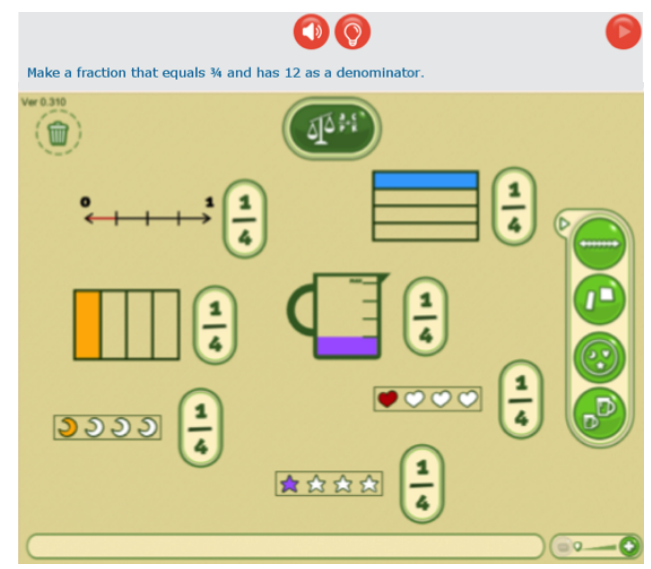

Fig. 1. Exploratory learning environment.

Figure 1 shows the Fractions Lab interface of the exploratory learning environment. The learning task is displayed at the top of the screen. Students are asked to solve the task by selecting a representation (from the right-hand side menu) which they manipulate in order to construct an answer to the given task.

\section{User study}

To investigate our research questions we conducted a user study in which students were aligned to either the affect-aware support condition or the non-affect aware support.

47 students took part in this study. These participants were all primary school students, aged between 8 and 10 years old, recruited from two schools in the UK.

Students were randomly allocated in either of the groups. In the affect condition $(\mathrm{N}=25)$ students were given access to the full iTalk2Learn system, which uses the student's affective state and their performance to determine the feedback type and its presentation. In the non-affect condition $(\mathrm{N}=22)$ students were given access to a version of the iTalk2Learn system in which feedback is based on the student's performance only. Students engaged with the iTalk2Learn system for 40 minutes according to the experimental condition, which included either the affect-aware or the non-affect-aware support. While students were engaging 


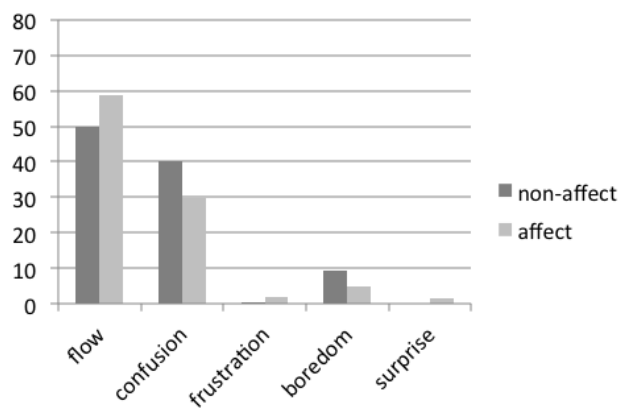

Fig. 2. Affective states of low/medium cognitive demanding tasks from the affect or non-affect condition.

with the iTalk2learn platform they were monitored from two researchers using the Baker-Rodrigo Ocumpaugh Monitoring Protocol [5]. The researchers who undertook the coding, and who were trained in the BROMP method, recorded the student affective states using the Human Affect Recording Tool (HART) Android mobile app.

The tasks provided to students differed in their cognitive demands as follows:

- low/medium cognitive demand: tasks where students are asked to create one or more fractions and check if they are equivalent in the compare box. Students can complete these tasks without performing any fraction calculation.

- high cognitive demand: tasks where students create a particular fraction as well as an equivalent fraction with particular constraints (e.g. a specific representation or a different denominator).

\section{Results}

To investigate our research questions we conducted a multivariate ANOVA using Pillais trace for the different affective states that occurred within the groups for the different learning tasks.

Tasks with low/medium cognitive demand The affective states of students while performing low/medium cognitive demanding tasks can be seen in Figure 2. It shows that students in the affect and non-affect condition were mainly in flow (affect mean: 58.91; non-affect mean: 49.91). There was no significant statistical difference in students' affective state based on which condition they were in $\mathrm{V}=0.086, \mathrm{~F}(5,67)=1.26, \mathrm{p}>.05$.

Tasks with high cognitive demand The affective states of students in high cognitively demanding tasks can be seen in Figure 3. It shows that in the affect condition students were mainly in flow (affect mean: 72.12; non-affect mean: 


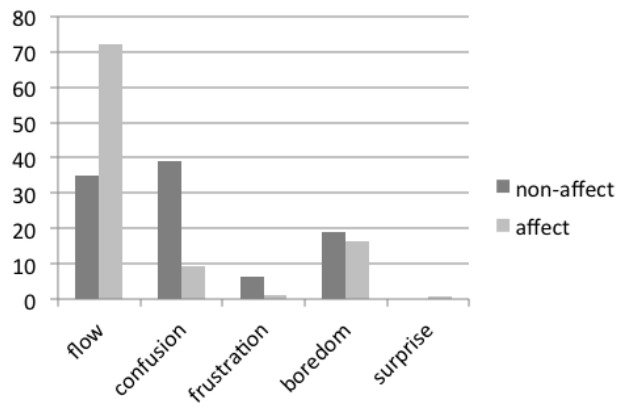

Fig. 3. Affective states of high cognitive demanding task from the affect or non-affect condition.

35.78). While students in the non-affect condition were mainly confused (affect mean: 9.21; non-affect mean: 39.08;). There was a significant statistical difference in students' affective state based on which condition they were assigned to $\mathrm{V}=0.29 \mathrm{~F}(4,64)=6.55, \mathrm{p}<.05$. Follow-up t-tests between the different affective states and the conditions, revealed a significant difference in students being in flow and the affect and non-affect condition $(\mathrm{t}(55.42)=3.92, \mathrm{p}<.05)$. Also, there was a significant difference between the groups and students being confused $(\mathrm{t}(45.52)=-4.12 . \mathrm{p}<.05)$.

\section{Discussion and Conclusion}

We developed a system that is able to provide intelligent support that takes into account students' affective state. The aim of the affect aware support is to move students from negative into positive affective states and hence tries to regulate a students' affective state by tailoring its feedback [3].

The intelligent affect-aware support (affect condition) was compared against support that was based on students performance only (non-affect condition). The results show that in low/medium cognitive demanding tasks no difference between the affect and non-affect group in students affective state was detected. However, on high cognitive demanding tasks there was a significant difference between students who received the affect-aware support and the students who received the support based on their performance only. Students in the affect condition were significantly more in flow and less confused than students in the non-affect condition. This may imply that when the cognitive demands of the task are high, students might find it difficult to regulate their affective states. However, when affect-aware support is provided in high cognitive demand tasks, students were able to regulate their affective states effectively and might have been moved from negative into positive affective states via the affect-aware support.

\section{Acknowledgments}

This research was funded by the European Union in the Seventh Framework Programme (FP7/2007-2013) in the iTalk-2Learn project (318051). 


\section{References}

1. Baker, R.S.J.d., D’Mello, S.K., Rodrigo, M.T., Graesser, A.C.: Better to be frustrated than bored: The incidence, persistence, and impact of learners cognitiveaffective states during interactions with three different computer-based learning environments. Int. J. Hum.-Comput. Stud. 68(4), 223241 (apr 2010)

2. D'Mello, S.K., Lehman, B., Pekrun, R., Graesser, A.C.: Confusion can be beneficial for learning. Learning \& Instruction 29(1), 153170 (2014)

3. Grawemeyer, B., Mavrikis, M., Holmes, W., Gutiérrez-Santos, S., Wiedmann, M., Rummel, N.: Affective learning: Improving engagement and enhancing learning with affect-aware feedback. User Modeling and User-Adapted Interaction - Special Issue on Impact of Learner Modeling (2017)

4. Kort,B.,Reilly,R.,Picard,R.: An affective model of the interplay between emotions and learning. In: IEEE International Conference on Advanced Learning Technologies. No. 43-46 (2001)

5. Ocumpaugh, J., Baker, R., Rodrigo, M.: Baker-rodrigo observation method protocol (bromp) 1.0. training manual version 1.0. Tech. rep., New York, NY: EdLab. Manila, Philippines: Ateneo Laboratory for the Learning Sciences. (2012)

6. Porayska-Pomsta, K., Mavrikis, M., Pain, H.: Diagnosing and acting on student affect: the tutors perspective. User Modeling and User-Adapted Interaction 18(1), $125173(2008)$ 